Research Article

\title{
A Satisficing Heuristic Decision-Making Model under Limited Attention and Incomplete Preferences
}

\author{
Xinlin $W u \mathbb{D}^{1,2}$ and Daoxin Ding $\mathbb{D}^{1,2}$ \\ ${ }^{1}$ Department of Mathematics, Hubei University of Education, Wuhan 430205, China \\ ${ }^{2}$ Institute of Big Data Modeling and Intelligent Computing, Hubei University of Education, Wuhan 430205, China \\ Correspondence should be addressed to Daoxin Ding; daoxinding@yeah.net
}

Received 12 September 2021; Revised 24 October 2021; Accepted 12 November 2021; Published 3 December 2021

Academic Editor: Jun Ye

Copyright ( 2021 Xinlin Wu and Daoxin Ding. This is an open access article distributed under the Creative Commons Attribution License, which permits unrestricted use, distribution, and reproduction in any medium, provided the original work is properly cited.

\begin{abstract}
Classical choice theory assumes that a decision-maker considers all feasible alternatives. However, a decision-maker in the real world can not consider all alternatives because of limited attention. In this paper, we propose a satisficing choice model to describe the choice procedure based on the incomplete preferences under the limited attention of the decision-maker. Moreover, the existence and rationality properties of the satisficing choice model on the different domains are studied combined with some proposed rationality conditions. Further, the proposed satisficing choice model is applied to a case of quality competition. Results show that the satisficing choice model of this paper is of a certain theoretical guiding significance to a kind of emergency decisions made by decision-makers under the circumstance of time pressure and limited information. It can also be the theoretical foundation for the study on the boundedly rational decision-making.
\end{abstract}

\section{Introduction}

Classical choice theory assumes that a decision-maker (DM) is fully rational. This implies that she perceives all the given alternatives before deciding, has infinite computational capabilities, and always chooses the best alternative from the set of alternatives according to an invariant preference relation. However, DMs in the real world do not perceive all the given alternatives before making their decisions, but typically discover and analyze alternatives sequentially in the process of decision-making. In other words, alternatives are presented to the DMs in a certain order (time or space order). For instance, after graduates send many resumes to different firms for a job, they may receive job offers sequentially in time. Because of various kinds of goods, consumers cannot remember and analyze all the goods in a short time while shopping online. They can only browse or choose goods in a certain order (from left to right or from top to bottom) in a web page. This assumption of full rationality ignores external environment and cognitive limitations of DMs, which has been criticized as unrealistic.
Many studies have been carried out in order to highlight the limitations of full rationality [1-3].

The most systematic attack to the assumption of full rationality is directed by Simon [4]. He pointed out that the DM in the real world cannot obtain all information, and has only finite computational capabilities. In addition, he argued that the structure of the environment plays a central role in permitting further simplifications of the assumption of full rationality. Simon criticizes the assumption that the DM perceives all alternatives before deciding. He argues that it would be more realistic to assume that the DM discovers and analyzes alternatives sequentially. When alternatives are examined sequentially, the DM may regard the first satisfactory alternative that is evaluated as such as the one actually selected. Simon [4] presented further the principle of satisficing heuristic, that is, individuals discover and analyze alternatives sequentially and stop searching as soon as they identify the first alternative that they judge to be satisfactory. In Simon's spirit, many studies have focused on deriving axioms and providing axiomatic perspectives of the satisficing heuristic. For example, Rubinstein and Salant [5] 
explored some axioms regarding choice functions from lists. Manzini and Mariotti [6] studied some properties of sequential rationalizability and provided a full characterization of choice functions rationalizable by two and three rationales. Salant and Rubinstein [7] developed a framework for modelling choice in the presence of framing effects. Caplin and Dean [8] characterized the nonstandard data on the evolution of provisional choices with contemplation time when the resulting data could have been generated by a general form of sequential search. Papi [9] proposed an axiomatic characterization of the principle of satisficing heuristic under various informational structures. Masatlioglu et al. [10] provided a choice theoretical foundation for maximizing a single preference relation under limited attention. Masatlioglu and Nakajima [11] formulated a framework to study behavioral search by utilizing the idea of consideration sets when the DM lack information or lack the cognitive capacity to alternatives. Lleras et al. [12] modelled individuals who might pay attention to only a subset of the choice problem presented to them. Manski [13] interpreted satisficing as a class of decision strategies that a person might use when deliberation is costly. Alonso and ülkü [14] characterized the resulting behavior using a single axiom without imposing any structure on how the consideration sets are formed. Kimya [15] provided a characterization of the procedure and illustrated how to identify the underlying preference and consideration set. Moreover, the stochastic satisficing models have been presented in many studies. For example, Manzini and Mariotti [16] assumed that the agent considers each feasible alternative with a given (unobservable) probability. They modelled a boundedly rational agent who suffers from limited attention. Aguiar et al. [17] developed necessary and sufficient conditions for stochastic choice data to be consistent with satisficing, assuming that preferences are fixed, but search order may change randomly. Brady and Rehbeck [18] examined the role of stochastic feasibility in consumer choice using a random conditional choice set rule and characterized the model from conditions on stochastic choice data. Aguiar [19] introduced a new stochastic choice rule that categorizes objects in order to simplify the choice procedure. He provided the necessary and sufficient conditions that characterize this model in a complete stochastic choice dataset. González-Valdés and de Dios Ortúzar [20] implemented the three principles of the Satisficing Theory mathematically. They obtained a discrete choice model in which the decision-maker chooses the first satisfactory alternative. Aguiar and Kimya [21] characterized Simon's search and satisficing model with an adaptive threshold and random search (SM-AT). The DM consistent with the SM-AT is endowed with a utility function, a random search distribution, and a deterministic but menu-dependent threshold. Caplin et al. [22] united two basic approaches to modelling limited attention in choice. They provided necessary and sufficient conditions for rationally inattentive behavior which allow the identification of consideration sets. Kovach and Ülkü [23] axiomatized a model of satisficing which features random thresholds and the possibility of choice abstention. Other studies have focused on designing experiments to test whether the DM's behavior is consistent with the satisficing heuristic of Simon. For example, Caplin et al. [24] developed a search-theoretic choice experiment to study the impact of incomplete consideration on the quality of choices. Reutskaja et al. [25] studied decisions that involve choosing between different numbers of options under time pressure using eye-tracking to monitor the search process of the subjects. Stüttgen et al. [26] conducted a visual conjoint experiment to collect search and choice data. They modelled search and product evaluation jointly and allowed for interdependence between them. Zhao and Huang [27] verified the boundedly rational route choice behavior by a laboratory experiment and proposed a novel model to formulate the behavior under satisficing rule. Hey et al. [28] tested a theory of Manski [13] by an experimental test and had a different way of generating imprecise information. Van Loo et al. [29] used eye-tracking measures to account for attribute nonattendance in choice experiments. Sandorf and Campbell [30] attempted to systematically explore the use of the satisficing heuristic in the context of a stated choice experiment. Caplin et al. [24] and Stüttgen et al. [26]'s experimental results showed that individual behavior is consistent with the satisficing heuristic. Reutskaja et al. [25]; Hey et al. [28] and Sandorf and Campbell's [30] experimental results showed that individual behavior is only partly consistent with the satisficing heuristic.

In the abovementioned study of the satisficing heuristic, a complete preference relation is a common assumption under the limited attention of the DM. The incompleteness of individual preferences is less considered. There are two major limitations in the abovementioned literatures. First, the rationality hypothesis of individual preferences is strong. The abovementioned literatures mainly considered a complete preference relation on the consideration set, and the DM can choose the best alternative. However, Simon [4] considered that a DM's cognitive limitations may to some degree prevent him from perceiving his own preferences among the available alternatives because of environmental complexity. This paper assumes that the DM's preferences are incomplete. The DM can only choose a satisficing alternative but not always the best alternative in the consideration set. Second, the probability of obtaining a satisfactory alternative is not high in a short time. A series of nested sets are used to describe the consideration set in the abovementioned literatures. This means that the number of alternatives in the DM's consideration set will increase with the extension of the search time. On the one hand, the increase of the number of alternatives in the consideration set will increase the difficulty for the DM to choose. On the other hand, the DM will constantly compare the newly added alternatives with the original ones, thus increasing the time for the DM to obtain a satisfactory alternative. In order to improve the efficiency of decisionmaking, a series of incompatible sets are used to describe the consideration set in this paper. This implies that it is not necessary to compare the newly added alternatives with all the original alternatives. Therefore, the DM can obtain a satisfactory alternative with a high probability in a short time. 
Under the circumstance of time constraint and limited information, the DM often want to quickly obtain a satisfactory alternative and cannot compare all alternatives. This means that the DM's preferences may not be complete. Recently, the decision-making based on incomplete preferences has received extensive attention in various fields $[31,32]$. Motivated by the challenge of remedying the abovementioned insufficient research on the satisficing heuristic and inspired by the work of Papi [9], this study proposes a satisficing heuristic decision-making model based on incomplete preferences under limited attention. The paper is organized as follows: Section 2 develops the formal model. Sections 3 and 4 develop and character the satisficing choice model under different conditions. Section 5 gives an application of the model. Section 6 summarizes this paper.

\section{The Model}

Let $X$ be a finite set of alternatives, where $\Omega$ represents the set of all nonempty subsets of $X$. In standard choice theory, a choice problem is simply a choice set $A \in \Omega$. In this framework, we define an extended choice problem as a pair $\left(A,\left\{A_{j}\right\}\right)$, where $A \in \Omega$ is a choice set and $\left\{A_{j}\right\}$ represents a sequence with which the DM examines the alternatives in $A$. We call $\left\{A_{j}\right\}$ subset sequence and define it as follows.

Definition 1. A subset sequence of the set $A \in \Omega$ is a sequence $\left\{A_{j}\right\}_{1}^{n}$ such that $A_{i} \cap A_{j}=\phi$, for all $i, j$ and $\cup_{i=1}^{n} A_{i}=A$, where each element $A_{i}$ is called a stage of the subset sequence $\left\{A_{j}\right\}$.

Definition 1 is similar to the definition of Papi [33]; the difference between them is that the subset sequence of Definition 1 is composed of disjoint sets, while that of Papi [33] is composed of nested sets. We consider that the assumption about disjoint search sets is more realistic in the real world. For example, a consumer is faced with large quantities of goods of one kind in different webpages in online purchases. In case of time pressure and incomplete information, if a consumer plans to purchase one from those goods in dozens of webpages, and she browses goods page after page, but would not like to go back to previously viewed webpages. We assume that these goods in one web page is considered to be a search set, and therefore, these search sets can be assumed to be disjoint.

Let an extended choice function be a choice function defined on the domain $D_{1}=\left\{\left(A,\left\{A_{j}\right\}\right) \mid A \in \Omega\right.$ and $\left\{A_{j}\right\}$ is a subset sequence of $\left.A\right\}$. We assume that $c\left(A,\left\{A_{j}\right\}\right)$ is nonempty and chooses one alternative from $A$. A (binary) relation on the set $X$ is a subset of $X \times X$. Let us write $P$ for the (asymmetry) binary relation on $X$ that encodes the DM's strict preferences among the alternatives. In this framework, we assume that the relation $P$ is acyclic and incomplete. We can define its complement $\bar{P}=\{(x, y) \mid(x P y)\}$. Let $x_{s} \in X$ be a satisfactory alternative which meets the minimum level of satisfaction of the DM. Let $G_{p}\left(A ; x_{s}\right)=\left\{x \in A \mid x_{s} \bar{P} x\right\}$ be the set of satisfactory alternatives in the set $A$. We assume that there always exist satisfactory alternatives in the choice set $A$, i.e., $G_{P}\left(A ; x_{s}\right) \neq \phi$. Let $\max (A ; P)=\{x \in A \mid \forall y \in A, y \bar{P} x\}$ be the set of maximal alternatives according to the relation $P$ in the set $A$. Since $P$ is assumed to be acyclic and incomplete, then the set $\max (A ; P)$ is not always a singleton.

Definition 2. (see Masatlioglu et al. [10]). $\Gamma$ is a mapping from $\Omega$ to $\Omega$ with $\phi \neq \Gamma(A) \subset A$ for every nonempty set $A \in \Omega, \Gamma(A)$ is called a consideration set.

Here, the notion of consideration sets is consistent with standard search theory (Masatlioglu et al. [10]; Masatlioglu and Nakajima [11]). A consideration set is the subset of all available alternatives to which the DM pays attention.

Definition 3. $c$ is a satisficing choice function on the domain $D_{1}$ if and only if there exist an acyclic and incomplete strict relation $P$, the minimum satisfactory alternative $x_{s} \in X$, and a consideration set $\Gamma(A) \subset A$ such that $c\left(A,\left\{A_{j}\right\}\right) \in$ $\max (\Gamma(A) ; P)$ where $\Gamma(A)=A_{j^{*}}$ and $j^{*}=\min \left\{j \mid A_{j} \cap\right.$ $\left.G_{\mathrm{P}}\left(A ; x_{s}\right) \neq \phi\right\}$.

Definition 3 implies that there always exists a satisfactory alternative in some stage of the sequence $\left\{A_{j}\right\}$. Here, a DM chooses an alternative based on the acyclic and incomplete strict relation $P$ and the minimum satisfactory alternative $x_{s}$. Specifically, if there exists an alternative which is not inferior to $x_{s}$, then it is considered to be satisfactory. The DM searches through the sequence $\left\{A_{j}\right\}$. If she identifies at least one satisfactory alternative in the first stage, then she stops searching at $A_{1}$, which becomes the consideration set, and chooses one satisfactory alternative in $A_{1}$. Otherwise, she continues to explore the second stage. This process is repeated until one satisfactory alternative is explored.

\section{The Decision Rationality Analysis under the Observable Search Order}

Suppose that we observe the DM making choices in the premise of the DM's limited attention and incomplete preferences. We are interested in identifying the conditions under which her behavior is consistent with the satisficing procedure. We explore these identification issues under two different domains. Under the full domain $D_{1}$, we assume that the search order of the sequence $\left\{A_{j}\right\}$ is observable, and the DM searches alternatives from the first stage $A_{1}$ of $\left\{A_{j}\right\}$. Now we move to the axiomatic characterization, some rationality conditions are introduced. The existence and rationality properties of a satisficing choice function on the domain $D_{1}$ are studied combined with these conditions.

Condition 1. $\quad x, y \in \Gamma(A) \cap \Gamma(B), x=c\left(A,\left\{A_{j}\right\}\right) \Rightarrow y \neq$ $c\left(B,\left\{B_{j}\right\}\right)$.

Condition 2. $x=c\left(A,\left\{A_{j}\right\}\right), y \in A \backslash\{x\} \Rightarrow x=c(A \backslash\{y\}$, $\left.\left\{A_{j}\right\}\right)$.

Condition 1 is a weakening of Weak Axiom Revealed Preference (Papi [33]), where $\left\{A_{j}\right\}$ and $\left\{B_{j}\right\}$ are two subset sequences of $A$ and $B$, respectively, and $\Gamma(A)$ and $\Gamma(B)$ are two consideration sets of $A$ and $B$, respectively. Condition 1 indicates that when both alternatives $x$ and $y$ are contained 
in two consideration sets $A$ and $B$, respectively, if $x$ is chosen in a sequence, then $y$ is not chosen in another sequence, which implies that if a consumer searches a satisfactory goods in one supermarket, and she will not go to another supermarket to buy another goods of the same kind. Condition 2 indicates that if $x$ is chosen in a sequence, then removing another alternative from this sequence does not affect a consumer's choice, which implies that removing irrelevant goods does not affect her choice.

Proposition 1. (1) If $c$ is a satisficing choice function on the domain $D_{1}$, then it satisfies Condition 1. (2) Let $c\left(A,\left\{A_{j}\right\}\right)$ be an extended choice function on the domain $D_{1}$, and $c\left(A,\left\{A_{j}\right\}\right) \in \max (A ; P)$. If $c\left(A,\left\{A_{j}\right\}\right)$ satisfies Condition 1 , then it is a satisficing choice function.

Proof

(1) The conclusion is obvious.

(2) Let $\left\{A_{j}\right\}$ be a subset sequence of $A \in \Omega$. We assume that $\Gamma(A)$ is a consideration set, and $\Gamma(A)=A_{1}=$ $\{x, y, z\}$. If $x P y$ and $y P z$, then $y \neq c\left(A,\left\{A_{j}\right\}\right)$, $z \neq c\left(A,\left\{A_{j}\right\}\right)$. If $z P x$, then $x \neq c\left(A,\left\{A_{j}\right\}\right)$, contradicting $c\left(A,\left\{A_{j}\right\}\right) \in A_{1}$. Hence, $P$ is acyclic and $x=c\left(A,\left\{A_{j}\right\}\right)$. This implies that $z \bar{P} x$, and hence, $P$ is incomplete. Let $\left\{B_{j}\right\}$ be a subset sequence of $B \in \Omega$, and $x, y \in \Gamma(A) \cap \Gamma(B)$. If $x=c\left(A,\left\{A_{j}\right\}\right)$, there exists another alternative $y \in \Gamma(A)$ such that $y P x$, and hence, $y=c\left(B,\left\{B_{j}\right\}\right)$, where $\Gamma(B)=B_{1}=$ $\{x, y\}$. Therefore, by Condition $1, x \neq c\left(A,\left\{A_{j}\right\}\right)$, contradicting $x=c\left(A,\left\{A_{j}\right\}\right)$. This implies that $\forall y \in \Gamma(A) \backslash\{x\}$ such that $y \bar{P} x$, that is $c\left(A,\left\{A_{j}\right\}\right) \epsilon$ $\max (A ; P)$. Let $x_{s} \in X$ be the minimum satisfactory alternative, and $\forall x \in \Gamma(A)$. Since $c\left(A,\left\{A_{j}\right\}\right) \epsilon$ $\max (A ; P)$, and hence, $c\left(A,\left\{A_{j}\right\}\right) \in G_{p}\left(A ; x_{s}\right)$, there exists a subset $A_{j} \subset A, j \in N$ such that $A_{j} \cap G_{\mathrm{P}}\left(A ; x_{s}\right) \neq \phi$. Therefore, we have $c(A$, $\left.\left\{A_{j}\right\}\right) \in A_{j^{*}}$, where $j^{*}=\min \left\{j \mid A_{j} \cap G_{P}\left(A ; x_{s}\right) \neq \phi\right\}$. So $x \in A_{j^{*}}$. Conversely, if $x \in A_{j^{*}}$, then we have $x \in \Gamma(A)$ since $c\left(A,\left\{A_{j}\right\}\right) \in G_{p}\left(A ; x_{s}\right)$. Therefore, $\Gamma(A)=A_{j^{*}}$.

Proposition 1 gives a condition about the existence of a satisficing choice function on the domain $D_{1}$. Moreover, Proposition 1 indicates that condition 1 can be regarded as a criterion of satisficing behavior.

Proposition 2. If $c$ is a satisficing choice function on the domain $D_{1}$, then it satisfies Condition 2.

Proof. Let $x=c\left(A,\left\{A_{j}\right\}\right)$ and $\Gamma(A)$ be a consideration set. Since $c$ is a satisficing choice function, and hence, $c\left(A,\left\{A_{j}\right\}\right) \in \max (A ; P) . \forall y \in \Gamma(A)$, we have $x=c(A \backslash\{y\}$,
$\left.\left\{A_{j}\right\}\right)$. Moreover, since $\Gamma(A) \subset A$ and $x=c\left(A,\left\{A_{j}\right\}\right)$, so $\forall y \in A \backslash \Gamma(A)$, we have $x=c\left(A \backslash\{y\},\left\{A_{j}\right\}\right)$.

Proposition 2 gives a rationality property of a satisficing choice function on the domain $D_{1}$.

Proposition 3. Let $c$ be a satisficing choice function on the domain $D_{1}$, (1) $x \in G_{p}\left(A ; x_{s}\right)$ if and only if $x \in A_{i} \subset A$ and there exists noy $\in A_{j} \subset A, j>i$ such that $c\left(A,\left\{A_{j}\right\}\right)=y$; (2) $x \in \Gamma(A)$ if and only if there exists some subset $A_{i}$ such that $x, y \in A_{i}$ and $y$ precedes $x, y=c\left(A,\left\{A_{j}\right\}\right)$; and (3) $x P y$ only if there exists some stage $A_{i}$ of a sequence $\left(A,\left\{A_{j}\right\}\right)$ such that $x=c\left(A,\left\{A_{j}\right\}\right)$ and $y \in \Gamma(A)=A_{i}$.

Proof

(1) $x \in G_{p}\left(A ; x_{s}\right)$ implies that $x$ is a satisfactory alternative in subset $A$, and hence, the DM stops searching after exploring the alternative $x$. So if $x \in A_{i} \subset A$, then there exists no $y \in A_{j} \subset A, j>i$ such that $c\left(A,\left\{A_{j}\right\}\right)=y$. Conversely, assume that $x \notin G_{p}\left(A ; x_{s}\right)$, this implies that there exists no satisfactory alternative in subset $A_{i}$. Since $G_{P}\left(A ; x_{s}\right) \neq \phi$, there exists another alternative $y \in A_{j} \subset A, j>i$ such that $c\left(A,\left\{A_{j}\right\}\right)=y$, contradicting the given condition.

(2) The conclusion is obvious.

(3) If $x P y$, then there exists some stage $A_{i}$ such that $\Gamma(A)=A_{i}=\{x, y\}$. And therefore, $x=c\left(A,\left\{A_{j}\right\}\right)$ since $c$ is a satisficing choice function.

Proposition 3 investigates the conditions under which we can infer from choices whether or not an alternative is revealed to be satisfactory, to be considered and to be preferred to another alternative on the domain $D_{1}$. Moreover, we can not judge whether an alternative is revealed to be preferred to another alternative under limited attention and incomplete preferences even if it is considered and chosen, which is different from classical revealed preference theory.

\section{The Decision Rationality Analysis under the Incompletely Observable Search Order}

In reality, it is hard to assume that the order with which the DM explores alternatives is observable. For example, consider a consumer who purchases goods online. The order with which she browses or chooses goods is uncertain usually. She may explore goods from left to right or from top to bottom in a web page, and so on. On the contrary, it seems more realistic to assume that the search order is incompletely observable. Therefore, the domain under which we study the issue is described as $D_{2}=\left\{\left(A, A_{k}\right) \mid A_{k} \subset\right.$ $\left.A \in \Omega, A_{k} \cup \bar{A}_{k}=A, k=1, \ldots, n\right\}$, where $A_{k}$ is some (observable) stage of the subset sequence, and the search orders of other stages $\bar{A}_{k}$ are assumed to be unobservable. 
Definition 4. $c$ is a satisficing choice function on the domain $D_{2}$ if and only if there exist an acyclic and incomplete relation $P$, the minimum satisfactory alternative $x_{s} \in X$, and a consideration set $\Gamma(A) \subset A$ such that $c\left(A, A_{k}\right) \in$ $\max (\Gamma(A) ; P)$, where $\Gamma(A)=\left\{\begin{array}{ll}A_{k} & G_{p}\left(A_{k} ; x_{s}\right) \neq \phi \\ \bar{A}_{k} & \text { otherwise }\end{array}\right.$.

Definition 4 is similar to Definition 3, and the difference between them is that the DM searches alternatives from some (observable) stage $A_{k}$, but not from the first stage $A_{1}$. Moreover, the search orders of other stages $\bar{A}_{k}$ are unobservable.

Similarly, the existence and rationality properties of a satisficing choice function on the domain $D_{2}$ are studied combined with the following Conditions 3 and 4 . Moreover, Conditions 3 and 4 on the domain $D_{2}$ are similar to Conditions 1 and 2 on the domain $D_{1}$, respectively.

Condition 3. $x, y \in \Gamma(A) \cap \Gamma(B), x=c\left(A, A_{k}\right) \Rightarrow y \neq c\left(B, B_{k}\right)$

Condition 4. $x=c\left(A, A_{k}\right), y \in A_{k} \backslash\{x\} \Rightarrow x=c\left(A, A_{k} \backslash\{y\}\right)$.

Proposition 4. (1) If $c$ is a satisficing choice function on the domain $D_{2}$, then it satisfies Condition 3. (2) Let $c\left(A, A_{k}\right)$ be an extended choice function on the domain $D_{2}$, and $c\left(A, A_{k}\right) \in \max (A ; P)$. If $c\left(A, A_{k}\right)$ satisfies Condition 3, then it is a satisficing choice function.

\section{Proof}

(1) The conclusion is obvious.

(2) Let $\left(A, A_{k}\right) \in D_{2}, \Gamma(A)=A_{k}=\{x, y, z\}$. If $x P y$ and $y P z$, then $z P x$. If $z P x$, then $x \neq c\left(A, A_{k}\right)$, contradicting $c\left(A, A_{k}\right) \in A_{k}$. Hence, $P$ is acyclic and $x=c\left(A, A_{k}\right)$. This implies that $z \bar{P} x$, and hence, $P$ is incomplete. Let $\left(B, B_{k}\right) \in D_{2}$ and $x, y \in \Gamma(A) \cap$ $\Gamma(B)$. If $x=c\left(A, A_{k}\right)$, there exists another alternative $y \in \Gamma(A)$ such that $y P z$, and hence, $y=c\left(B, B_{k}\right)$, where $\Gamma(B)=B_{k}=\{x, y\}$. Therefore, by Condition 3, $x \neq c\left(A, A_{k}\right)$, contradicting $x=c\left(A, A_{k}\right)$. This implies that $\forall y \in \Gamma(A) \backslash\{x\}$ such that $y \bar{P} x$, that is $c\left(A, A_{k}\right) \in \max (\Gamma(A) ; P)$. Let $x_{s} \in X$ be the minimum satisfactory alternative, and $\forall x \in \Gamma(A)$. Since $c\left(A, A_{k}\right) \in \max (\Gamma(A) ; P)$ and if $G_{P}\left(A_{k} ; x_{s}\right) \neq \phi$, we have $c\left(A, A_{k}\right) \in G_{\mathrm{p}}\left(A_{k} ; x_{s}\right), \quad c\left(A, A_{k}\right) \in A_{k}, \quad$ and hence, $x \in A_{k}$. Conversely, if $x \in A_{k}$ then we have $x \in \Gamma(A)$ since $c\left(A, A_{k}\right) \in G_{\mathrm{p}}\left(A_{k} ; x_{s}\right)$. Therefore, $\Gamma(A)=A_{k}$. If $G_{P}\left(A_{k} ; x_{s}\right)=\phi$, then we have $\Gamma(A)=\bar{A}_{k}$.

Proposition 4 proves the existence of a satisficing choice function on the domain $D_{2}$ when the search order is incompletely observable.

Proposition 5. If $c$ is a satisficing choice function on the domain $\mathrm{D}_{2}$, then it satisfies Condition 4

Proof. Let $x=c\left(A, A_{k}\right)$ and $\Gamma(A)$ be a consideration set. If $\Gamma(A)=A_{k}, \quad \forall y \in \Gamma(A), \quad$ we have $\quad x=c\left(A, A_{k} \backslash\{y\}\right)$
$\operatorname{sincec}\left(A, A_{k}\right) \in \max (\Gamma(A) ; P)$. If $\Gamma(A)=\bar{A}_{k}, \forall y \in A \backslash \Gamma(A)$, we have $x=c\left(A, A_{k} \backslash\{y\}\right)$.

Proposition 5 gives the rationality property of a satisficing choice function on the domain $D_{2}$ when the search order is incompletely observable.

Proposition 6. Suppose that $c$ is a satisficing choice function on the domain $D_{2},(1) x \in G_{p}\left(A ; x_{s}\right)$ if and only if there exists no $\left(A, A_{k}\right) \in D_{2}$ such that $x \in A_{k}$ and $c\left(A, A_{k}\right) \notin A_{k}$. (2) $x \in \Gamma(A)$ if there exists another alternative $y=c\left(A, A_{k}\right)$ and $y$ precedes $x$ in $A_{k}$. (3) $x P y$ only if there exists $\left(A, A_{k}\right) \in D_{2}$ such that $x=c\left(A, A_{k}\right)$ and $y \in A_{k}$.

Proof

(1) If $x \in G_{p}\left(A ; x_{s}\right)$, we assume that there exists $\left(A, A_{k}\right) \in D_{2}$ such that $x \in A_{k}$ and $c\left(A, A_{k}\right) \notin A_{k}$. Since $c\left(A, A_{k}\right) \in G_{p}\left(A ; x_{s}\right)$, then $\Gamma(A)=\bar{A}_{k}$. This implies that $G_{p}\left(A_{k} ; x_{s}\right)=\phi$, contradicting $x \in G_{p}\left(A ; x_{s}\right) \cap A_{k}$. Conversely, assume that $x \notin G_{p}\left(A ; x_{s}\right)$. Let $y \in \max (\Gamma(A) ; P)$, there exists $\left(A, A_{k}\right) \in D_{2}$ such that $x \in A_{k}$ and $y \in A \backslash A_{k}$. And hence, $y \in G_{p}\left(A ; x_{s}\right)$; this implies that $c\left(A, A_{k}\right)=y_{k} \notin A_{k}$.

(2) The conclusion is obvious.

(3) If $x P y$, then there exists $\left(A, A_{k}\right) \in D_{2}$ such that $A=\{x, y\}=A_{k}$. And hence $A_{k}=A=\Gamma(A)$. We have $x=c\left(A, A_{k}\right)$ since $c\left(A, A_{k}\right) \in \max (\Gamma(A) ; P)$.

Similar to Proposition 3, Proposition 6 investigates the conditions under which we can infer from choices whether or not an alternative is revealed to be satisfactory, to be considered and to be preferred to another alternative on the domain $D_{2}$.

\section{Application}

We first introduce some notations and definitions. Let $M$ be a finite set of all products $m \in M$. The market is composed of many firms that produce homogeneous products, and they have to decide simultaneously which product to produce. Let $N=\{1,2, \ldots, n\}$ be the set of firms (players), where $n \geq 2$ is the number of firms. The competition of firms is the competition of the product quality. Let $\sigma_{i}=\left\{\sigma_{i, m_{1}}, \ldots, \sigma_{i, m_{n}}\right\}$ be firm $i$ 's set of strategies, with $i \in N$. A firm $i$ s pure strategy is denoted by $\sigma_{i, m_{i}} \in \sigma_{i}$. A strategy profile of all firms is a vector $\sigma=\left(\sigma_{1, m_{1}}, \ldots, \sigma_{n, m_{n}}\right) \in \prod_{i=1}^{n} \sigma_{i}$, and the firm $i$ 's profit is denoted by $\pi_{i}(\sigma)$. Let $c(m)$ be the cost of producing one product $m$ and $p(m)$ be the price of the product $m$, and we assume $p(m)>n c(m)$. We assume that low quality is always associated with lower prices and high quality with higher prices. The goal of firms is to maximize profit.

We assume the consumers can be either fully rational consumers (FRCs) or boundedly rational consumers (BRCs). Let $R$ be a binary relation on $M$ representing a consumer's preferences, and $P$ and $I$ are the asymmetrical and symmetrical part of $R$, respectively. Let $m^{\max } \in\left\{m \in M \mid \bar{\exists} m^{\prime} \in M, m^{\prime} P m\right\}$ be the highest-quality product in $M$ and let $m^{\min } \in\left\{m \in M \mid \bar{\exists} m^{\prime} \in M, m P m^{\prime}\right\}$ be 
the lowest-quality product in $M$. Let $m^{s} \in M$ be the lowestsatisfactory product in $M$. A FRC's preferences are complete, and therefore, she always searches for the best product. However, a BRC's preferences are incomplete, and she cannot always search for the best product. The BRC searches for a satisfactory product. All products that are not inferior to $m^{s}$ are considered to be satisfactory for the BRC. The process is as follows:

(1) All firms decide to supply products meanwhile, and each firm supplies a product of the same quality

(2) The products supplied by the $n$ firms are ordered according to a certain order

(3) The consumer chooses a product

5.1. Equilibrium Analysis under Full Rationality. Assume first that firms know that the consumer is a FRC. If the consumer is fully rational, then she is an expected utility maximizer. We assume that the higher the quality of one product which the FRC chooses, the greater her utility. She chooses the best product according to her preference relation, and if products are of the same quality, then she chooses randomly.

Given a strategy profile $\sigma$, let $n(i, \sigma)=\left|\left\{j \in N \mid m_{j} I m_{i}\right\}\right|$ be the number of firms that supply a product $m_{j}$ of the same quality as $m_{i}$. Firm $i$ 's expected profits depend on how many firms supply products of the same quality as $m_{i}$. For instance, if $m_{i} P m_{j}$ for all $j \in N$, then $n(i, \sigma)=1$ and $\pi_{i}(\sigma)=$ $p\left(m_{i}\right)-c\left(m_{i}\right)$. On the other hand, if $m_{i} I m_{j}$ for all $j \in N$, then $n(i, \sigma)=n$ and $\pi_{i}(\sigma)=(1 / n) p\left(m_{i}\right)-c\left(m_{i}\right)$. When $1<n(i, \sigma)<n, \quad \pi_{i}(\sigma)=(1 / n(i, \sigma)) p\left(m_{i}\right)-c\left(m_{i}\right)$. Proposition 7 characterizes the equilibrium of the $n$-firm game, where the consumer is fully rational.

Proposition 7. In the n-firm game in which the consumer is fully rational, there exists a pure-strategy Nash equilibrium. In some conditions, the Nash equilibrium's solution is unique, i.e., $\sigma^{\max }=\left(\sigma_{1, m^{\max }}, \ldots, \sigma_{n, m^{\max }}\right)$.

Proof. We first prove existence. Assume that all firms play $\sigma^{\max }=\left(\sigma_{i, m^{\max }}\right)_{i \in N}$. In this case, each firm's expected profits are $(1 / n) p\left(m^{\max }\right)-c\left(m^{\max }\right)$. Suppose by contradiction that $\sigma^{\max }$ is not a Nash equilibrium. Assume that firm $i$ deviates, by playing some pure strategy $m^{\prime}$ and $m^{\max } \mathrm{Pm}^{\prime}$. In this case, firm i's expected profits are $-c\left(m^{\prime}\right)$. Obviously, firm i's expected profits become smaller by the deviation, and $\sigma^{\max }$ is a Nash equilibrium. Next, we prove uniqueness. Suppose, by contradiction, that there is another pure-strategy Nash equilibrium $\sigma^{\prime}=\left(\sigma_{i, m^{\prime}}\right)_{i \in N}$ different from $\sigma^{\max }$. In this case, each firm's expected profits are $(1 / n) p\left(m^{\prime}\right)-c\left(m^{\prime}\right)$. Assume that firm $i$ deviates, by playing some pure strategy $m^{\prime \prime} P m^{\prime}$ and $m^{\prime \prime} P m^{\prime}$. In this case, firm i's expected profits are $p\left(m^{\prime \prime}\right)-c\left(m^{\prime \prime}\right)$. If $p\left(m^{\prime \prime}\right)-c\left(m^{\prime \prime}\right) \geq(1 / n) p\left(m^{\prime}\right)-c\left(m^{\prime}\right)$, then firm i's expected profits become greater by the deviation. Therefore, $\sigma^{\prime}$ is not an equilibrium. Hence, $\sigma^{\max }$ is unique.

Proposition 7 indicates that all firms should supply the FRC with the highest-quality product in order to maximize their expected profits. In this case, the FRC can obtain the maximal utility. Moreover, if all firms supply a low-quality product $m^{\prime}$ and $m^{\max } \mathrm{Pm}^{\prime}$ under the condition $n p\left(m^{\max }\right)-p\left(m^{\prime}\right) \geq n\left(c\left(m^{\max }\right)-c\left(m^{\prime}\right)\right)$, firms can also obtain profits. However, the FRC cannot choose the highestquality product. It implies that there also exists an equilibrium although all firms do not supply the highest-quality product.

The game of Figure 1 represents the 2-firm game in which the consumer is a FRC, there are two firms, and $M=\left\{m^{\max }, m^{\min }\right\} . \quad$ Let $\quad c\left(m^{\min }\right)=20, p\left(m^{\min }\right)=100$, $c\left(m^{\max }\right)=50, p\left(m^{\max }\right)=200$.

The row player is firm 1 and the column player is firm 2 . The set of strategies is $\sigma=\left\{\left(\sigma_{1, m^{\min }}, \sigma_{2, m^{\min }}\right),\left(\sigma_{1, m^{\min }}, \sigma_{2, m^{\max }}\right)\right.$, $\left.\left(\sigma_{1, m^{\max }}, \sigma_{2, m^{\min }}\right),\left(\sigma_{1, m^{\max }}, \sigma_{2, m^{\max }}\right)\right\}$ and $m^{\max } \mathrm{Pm}^{\min }$. For instance, suppose that firm 1 plays $m^{\max }$ and firm $2 \mathrm{~m}^{\mathrm{min}}$. Since $m^{\max } P m^{\min }$, then the FRC buys $m^{\max }$. Therefore, firm 1's profits are 150 and firm 2's profits are -20 . Suppose that firm 1 plays $m^{\max }$ and firm $2 m^{\max }$, since $m^{\max } I m^{\max }$, the FRC buys $m^{\max }$ randomly. Therefore, firm 1 and firm 2 's expected profits are all 50 . The strategy profile $\left(\sigma_{1, m^{\max }}, \sigma_{2, m^{\max }}\right)$ is the Nash equilibrium of pure strategy in Figure 1 . When $2 p\left(m^{\max }\right)-p\left(m^{\min }\right) \geq 2\left(c\left(m^{\max }\right)-c\left(m^{\min }\right)\right)$, the strategy profile $\left(\sigma_{1, m^{\min }}, \sigma_{2, m^{\min }}\right)$ is also the Nash equilibrium.

\subsection{Equilibrium Analysis under Bounded Rationality.} Assume now that firms know that the consumer is a BRC. If the consumer is a $\mathrm{BRC}$, she cannot compare all products under limited attention. We assume that the BRC follows the satisficing heuristic, that is, she examines products sequentially and stops searching as soon as she identifies the first satisfactory product. Here, a set of products which contains the first satisfactory product of BRC's is called a consideration set. And then, the BRC chooses a satisfactory product only in a consideration set. When there are several satisfactory products in the consideration set, then she chooses randomly. For instance, assume that some firm that precedes firm $i$ supplies a satisfactory product. In this case, the BRC does not even consider firm $i$ 's product because she stops searching before encountering it. Unlike the FRC, if all products are discovered, that are not inferior to $\mathrm{m}^{s}$, then the BRC chooses randomly.

Next, we analyze the firm $i$ 's profits. If only the product $m_{i}$ provided by the firm $i$ is satisfactory to the BRC in the consideration set, then the firm $i$ 's profits are $p\left(m_{i}\right)-c\left(m_{i}\right)$. If products provided by $n(i, \sigma)(1<n(i, \sigma)<n)$ firms are all satisfactory to the BRC in the consideration set, then firm $i$ 's profits are $(1 / n(i, \sigma)) p\left(m_{i}\right)-c\left(m_{i}\right)$. Proposition 8 characterizes the equilibrium of the $n$-firm game, where the consumer is boundedly rational.

Proposition 8. In the n-firm game in which the consumer is boundedly rational, if $\forall m, m^{\prime} \in M$ and $m P m^{\prime} R m^{s}$ such that $p(m)-p\left(m^{\prime}\right) \leq n\left(c(m)-c\left(m^{\prime}\right)\right)$, then there exists a unique pure-strategy Nash equilibrium, and the Nash equilibrium's solution is $\sigma^{s}=\left(\sigma_{1, m^{s}}, \ldots, \sigma_{n, m^{s}}\right)$.

Proof. We first prove existence. Assume that all firms play $\sigma^{s}=\left(\sigma_{i, m^{s}}\right)_{i \in N^{*}}$. In this case, each firm's expected profits are 
Firm 2

\begin{tabular}{cc|c|c|} 
& & $\sigma_{2, m^{\min }}$ & $\sigma_{2, m^{\max }}$ \\
\cline { 3 - 3 } & $\sigma_{1, m^{\min }}$ & 30,30 & $-20,150$ \\
\cline { 2 - 3 } & $\sigma_{1, m^{\max }}$ & $150,-20$ & 50,50 \\
\cline { 3 - 4 } & &
\end{tabular}

FIGURE 1: Competitive strategy under full rationality for $n=2$ and $M=\left\{m^{\max }, m^{\min }\right\}$.

$\pi\left(\sigma^{s}\right)=(1 / n)\left(p\left(m^{s}\right)-c\left(m^{s}\right)\right)-((n-1) / n) c\left(m^{s}\right)$. Suppose by contradiction that $m^{\prime}$ is not a Nash equilibrium. If this is the case, then there must be at least one profitable deviation. Assume first that firm $i$ deviates, by playing some pure strategy $m^{\prime}$ such that $m^{\prime} P m^{s}$. In this case, firm i's expected profits are

$$
\begin{aligned}
\pi_{i}\left(\sigma_{i, m^{\prime}}, \sigma_{-i, m^{s}}\right) & =\frac{1}{n}\left(p\left(m^{\prime}\right)-c\left(m^{\prime}\right)\right)-\frac{n-1}{n} c\left(m^{\prime}\right) \\
& =\frac{1}{n} p\left(m^{\prime}\right)-c\left(m^{\prime}\right) .
\end{aligned}
$$

Since $m^{\prime} P m^{s}$ and $p\left(m^{\prime}\right)-p\left(m^{s}\right) \leq n\left(c\left(m^{\prime}\right)-c\left(m^{s}\right)\right)$, hence $\pi\left(\sigma^{s}\right) \geq \pi_{i}\left(\sigma_{i, m^{\prime}}, \sigma_{-i, m^{s}}\right)$, this implies that the deviation is not profitable. Next, suppose that firm $i$ deviates, by playing some pure strategy $m^{\prime \prime}$ such that $m^{s} P m^{\prime \prime}$. In this case, firm i's expected profits are $\pi_{i}\left(\sigma_{i, m^{\prime \prime}}, \sigma_{-i, m^{s}}\right)=-c\left(m^{\prime \prime}\right)$, which means that the deviation is also not profitable. Therefore, $\sigma^{s}$ is a Nash equilibrium.

Now we prove uniqueness. Suppose, by contradiction, that there is another pure-strategy Nash equilibrium $\sigma^{*}=$ $\left(\sigma_{i, m^{*}}\right)_{i \in N}$ different from $\sigma^{s}$. Assume that firms play $\sigma^{*}$, and hence, each firm $i^{\prime}$ s profits are $\pi\left(\sigma^{*}\right)=(1 / n) p$ $\left(m^{*}\right)-c\left(m^{*}\right)$. Suppose first that $m^{s} P m^{*}$, then each firm $i$ could profitably deviate by playing $m^{* *} P m^{*}$, and hence, $\sigma^{*}$ cannot be a Nash equilibrium. Next, suppose that $m^{*} P m^{s}$, if each firm $i$ deviates, by playing some pure strategy $m^{\prime \prime \prime}$ such that $m^{*} P m^{\prime \prime \prime} m^{s}$, and then, firm $i^{\prime}$ s expected profits are $\pi_{i}\left(\sigma_{i, m^{\prime \prime \prime}}, \sigma_{-i, m^{*}}\right)=(1 / n) p_{\prime \prime \prime}\left(m^{\prime \prime \prime}\right)-c\left(m^{\prime \prime \prime}\right)$. Since $p\left(m^{*}\right)-$ $p\left(m^{\prime \prime}\right) \leq n\left(c\left(m^{*}\right)-c\left(m^{\prime \prime \prime}\right)\right)$, hence $\pi\left(\sigma^{*}\right) \leq \pi_{i}\left(\sigma_{i, m^{\prime \prime \prime}}, \sigma_{-i, m^{*}}\right)$, this implies that the deviation to $m^{\prime \prime \prime}$ is profitable. Therefore, $\sigma^{*}$ cannot be a Nash equilibrium. Hence, $m^{s}$ is unique.

Proposition 8 indicates that all firms only supply the BRC with the minimal satisfactory product $m^{s}$ in order to maximize their expected profits under certain conditions. In this case, each firm $i^{\prime} s$ expected profits are $(1 / n) p\left(m^{s}\right)-c\left(m^{s}\right)$. The fact that the BRC follows the satisficing heuristic attenuates the mechanism of competition and induces firms not to supply the highest-quality product.

The game of Figure 2 represents the 2 -firm game in which the consumer is a BRC, there are two firms, and $M=\left\{m^{\max }, m^{s}\right\}$. Let $c\left(m^{s}\right)=35, p\left(m^{s}\right)=150, c\left(m^{\max }\right)=50$, $p\left(m^{\max }\right)=200$.

The row player is firm 1 and the column player is firm 2 . The set of strategies is $\sigma=\left\{\left(\sigma_{1, m^{s}}, \sigma_{2, m^{s}}\right), \quad\left(\sigma_{1, m^{s}}, \sigma_{2, m^{\max }}\right)\right.$, $\left.\left(\sigma_{1, m^{\max }}, \sigma_{2, m^{s}}\right),\left(\sigma_{1, m^{\max }}, \sigma_{2, m^{\max }}\right)\right\}$ and $m^{\max } R m^{s}$. It should be noted that their choices of FRCs and BRCs are different in face of products $m^{\max }$ and $m^{s}$.

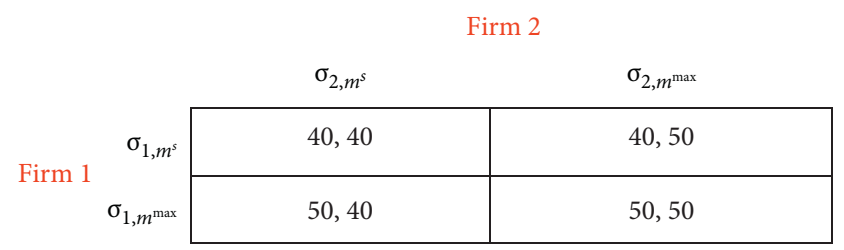

FIgURE 2: Competitive strategy under bounded rationality for $n=2$ and $M=\left\{m^{\max }, m^{s}\right\}$.

For instance, suppose that firm 1 plays $m^{s}$ and firm 2 $m^{\max }$. Since $m^{\max } R m^{s}$, then the FRC buys $m^{\max }$. Therefore, firm 1's profits are -35 and firm 2's profits are 150 . However, the products $m^{\max }$ and $m^{s}$ are indifferent $\left(m^{\max } I m^{s}\right)$ for the BRC, and hence, the BRC buys $m^{\max }$ or $m^{s}$ randomly. Therefore, firm 1 and firm 2's expected profits are 40 and 50, respectively. Moreover, the Nash equilibrium does not always exist, but only exists under a certain condition in Figure 2. For instance, when the condition $p\left(m^{\max }\right)-$ $p\left(m^{s}\right) \leq 2\left(c\left(m^{\max }\right)-c\left(m^{s}\right)\right)$ holds, the strategy profile $\left(\sigma_{1, m^{s}}, \sigma_{2, m^{s}}\right)$ is the Nash equilibrium.

\section{Conclusions}

This paper proposes a satisficing choice model in which the DM's behavior is consistent with the satisficing heuristic. Our main contribution is that we propose a satisficing choice rule in the premise of the DM's limited attention and incomplete preferences. We axiomatically characterize the proposed model under two different choice domains. In the application of quality competition, we characterize the equilibrium of the $n$-firm game, where the consumer can be either a FRC or a BRC. Moreover, an example of numerical analysis in case of the 2-firm game is given. Different from existing researches, the consideration set is established by disjoint sets to describe the limited attention of the DM in the premise of the DM's incomplete preferences in this paper. The satisficing choice model of this paper is to ensure that the DM is able to obtain a satisficing alternative in a short time with a larger probability. However, it is difficult for the DM to obtain the optimal alternative in the satisficing choice model.

Moreover, we point out the following research directions for future studies:

(1) The stochastic satisficing models (such as Caplin et al. [22]; Kovach and Ülkü [23]) have recently been a research hotspot. How to propose a stochastic satisficing choice rule in the premise of the DM's limited attention and incomplete preferences is worth studying further.

(2) It is also interesting to extend the satisficing choice model to consensus reaching for group decisionmaking settings (such as Zhang et al. [34]; Zhang et al. [35]).

\section{Data Availability}

No data were used to support this study. 


\section{Conflicts of Interest}

The authors declare that they have no conflicts of interest.

\section{Acknowledgments}

This work was supported by the Natural Science Foundation of Hubei Province (Grant no. 2019CFB782).

\section{References}

[1] J. Conlisk, "Why bounded rationality?" Journal of Economic Literature, vol. 34, no. 2, pp. 669-700, 1996.

[2] J. Lorkowski and V. Kreinovich, Bounded Rationality in Decision Making under Uncertainty: Towards Optimal Granularity, Cham: Springer International Publishing, New York, NY, USA, 2018.

[3] Y. Liu, H. Zhang, Y. Wu, and Y. Dong, "Ranking range based approach to madm under incomplete context and its application in venture investment evaluation," Technological and Economic Development of Economy, vol. 25, no. 5, pp. 877899, 2019.

[4] H. A. Simon, "A behavioral model of rational choice," Quarterly Journal of Economics, vol. 69, no. 1, pp. 99-118, 1955.

[5] A. Rubinstein and Y. Salant, "A model of choice from lists," Theoretical Economics, vol. 1, no. 1, pp. 3-17, 2006.

[6] P. Manzini and M. Mariotti, "Sequentially rationalizable choice," The American Economic Review, vol. 97, no. 5, pp. 1824-1839, 2007.

[7] Y. Salant and A. Rubinstein, "(A,f): choice with Frames," The Review of Economic Studies, vol. 75, no. 4, pp. 1287-1296, 2008.

[8] A. Caplin and M. Dean, "Search, choice, and revealed preference," Theoretical Economics, vol. 6, no. 1, pp. 19-48, 2011.

[9] M. Papi, "Satisficing choice procedures," Journal of Economic Behavior \& Organization, vol. 84, no. 1, pp. 451-462, 2012.

[10] Y. Masatlioglu, D. Nakajima, and E. Y. Ozbay, "Revealed attention," The American Economic Review, vol. 102, no. 5, pp. 2183-2205, 2012.

[11] Y. Masatlioglu and D. Nakajima, "Choice by iterative search," Theoretical Economics, vol. 8, no. 3, pp. 701-728, 2013.

[12] J. S. Lleras, Y. Masatlioglu, D. Nakajima, and E. Y. Ozbay, "When more is less: limited consideration," Journal of Economic Theory, vol. 170, no. 7, pp. 70-85, 2017.

[13] C. F. Manski, "Optimize, satisfice, or choose without deliberation? A simple minimax-regret assessment," Theory and Decision, vol. 83, no. 2, pp. 155-173, 2017.

[14] A. Alonso and L. ülkü, "Luce rule with limited consideration," Mathematical Social Sciences, vol. 93, no. 5, pp. 52-56, 2018.

[15] M. Kimya, "Choice, consideration sets, and attribute filters," American Economic Journal: Microeconomics, vol. 10, no. 4, pp. 223-247, 2018.

[16] P. Manzini and M. Mariotti, "Stochastic choice and consideration sets," Econometrica, vol. 82, no. 3, pp. 1153-1176, 2014.

[17] V. H. Aguiar, M. J. Boccardi, and M. Dean, "Satisficing and stochastic choice," Journal of Economic Theory, vol. 166, no. 6, pp. 445-482, 2016.

[18] R. L. Brady and J. Rehbeck, "Menu-dependent stochastic feasibility," Econometrica, vol. 84, no. 3, pp. 1203-1223, 2016.

[19] V. H. Aguiar, "Random categorization and bounded rationality," Economics Letters, vol. 159, no. 10, pp. 46-52, 2017.
[20] F. González-Valdés and J. d. D. Ortúzar, "The stochastic satisficing model: a bounded rationality discrete choice model," Journal of Choice Modelling, vol. 27, no. 2, pp. 74-87, 2018.

[21] V. H. Aguiar and M. Kimya, "Adaptive stochastic search," Journal of Mathematical Economics, vol. 81, no. 3, pp. 74-83, 2019.

[22] A. Caplin, M. Dean, and J. Leahy, "Rational inattention, optimal consideration sets, and stochastic choice," The Review of Economic Studies, vol. 86, no. 3, pp. 1061-1094, 2019.

[23] M. Kovach and L. Ülkü, "Satisficing with a variable threshold," Journal of Mathematical Economics, vol. 87, no. 3, pp. $67-76,2020$.

[24] A. Caplin, M. Dean, and D. Martin, "Search and satisficing," The American Economic Review, vol. 101, no. 7, pp. 28992922, 2011.

[25] E. Reutskaja, R. Nagel, C. F. Camerer, and A. Rangel, "Search dynamics in consumer choice under time pressure: an eyetracking study," The American Economic Review, vol. 101, no. 2, pp. 900-926, 2011.

[26] P. Stüttgen, P. Boatwright, and R. T. Monroe, "A satisficing choice model," Marketing Science, vol. 31, no. 6, pp. 878-899, 2012.

[27] C.-L. Zhao and H.-J. Huang, "Experiment of boundedly rational route choice behavior and the model under satisficing rule," Transportation Research Part C: Emerging Technologies, vol. 68, no. 7, pp. 22-37, 2016.

[28] J. D. Hey, Y. Permana, and N. Rochanahastin, "When and how to satisfice: an experimental investigation," Theory and Decision, vol. 83, no. 3, pp. 337-353, 2017.

[29] E. J. Van Loo, R. M. Nayga, D. Campbell, H.-S. Seo, and W. Verbeke, "Using eye tracking to account for attribute nonattendance in choice experiments," European Review of Agricultural Economics, vol. 45, no. 3, pp. 333-365, 2018.

[30] E. D. Sandorf and D. Campbell, "Accommodating satisficing behaviour in stated choice experiments," European Review of Agricultural Economics, vol. 46, no. 1, pp. 133-162, 2019.

[31] H. Zhang, Y. Dong, J. Xiao, F. Chiclana, and E. HerreraViedma, "Personalized individual semantics-based approach for linguistic failure modes and effects analysis with incomplete preference information," IISE Transactions, vol. 52, no. 11, pp. 1275-1296, 2020.

[32] J. Xiao, X. Wang, and H. Zhang, "Exploring the ordinal classifications of failure Modes in the reliability management: an optimization-based consensus model with bounded confidences," Group Decision and Negotiation, Springer, Berlin, Germany, 2021.

[33] M. Papi, Essays on Boundedly Rational Decision-Making: Theory, Applications, and Experiments, Doctoral thesis, University of Trento, Trento, Italy, 2011.

[34] Z. Zhang, Y. Gao, and Z. Li, "Consensus reaching for social network group decision making by considering leadership and bounded confidence," Knowledge-Based Systems, vol. 204, no. 9, Article ID 106240, 2020.

[35] Z. Zhang, Z. Li, and Y. Gao, "Consensus reaching for group decision making with multi-granular unbalanced linguistic information: a bounded confidence and minimum adjustment-based approach," Information Fusion, vol. 74, no. 10, pp. 96-110, 2021. 